\title{
Alternativas para el aprovechamiento del proceso investigativo en la enseñanza de la música
}

\section{Alternatives to exploiting the research process in benefit of music teaching}

JUAN-CARVAJAL, Mara Lioba†*, JUAN-CARVAJAL, Dargen Tania, SÁNCHEZ-USÓN, María José y VDOVINA, María

Universidad Autónoma de Zacatecas

Universidad Tecnológica de La Habana "José A. Echeverría”

ID $1^{\text {er }}$ Autor: Mara Lioba, Juan-Carvajal / ORC ID: 0000-0001-6968-3813, Researcher ID Thomson: P-7756-2016, CVU CONACYT ID: 216443

ID $1^{\text {er }}$ Coautor: Dargen Tania, Juan Carvajal / OCR ID: 0000-0002-8281-8169, Researcher ID Thomson: V-7816-2019

ID $2^{\mathrm{do}}$ Coautor: María José, Sánchez-Usón / ORC ID: 0000-0002-3409-4055, Researcher ID Thomson: S-7908-2018, CVU CONACYT ID: 432522

ID $3^{\text {er }}$ Coautor: María, Vdovina / ORC ID: 0000-0001-6656-0789, Researcher ID Thomson: S-7917-2018

DOI: $10.35429 /$ JPDL.2019.16.5.10.19

Recibido 29 de Junio 2019; Aceptado 30 Septiembre, 2019

\begin{abstract}
Resumen
La enseñanza artística conlleva hoy un esfuerzo superior respecto al desplegado en décadas anteriores. Sin demeritar calidad y rigor, el estudiante, el grupo y el docente compartían entonces, gradualmente, ideas, recursos, técnica y emociones dentro y fuera del contexto escolar. En la actualidad, el proceso se acelera; el número considerable de investigaciones en, sobre y para el arte, unido al impacto tecnológico y a la existencia de redes sociales que facilitan el intercambio, estimula contrastes informativos que asignan nuevos retos al docente, como fomentar la creatividad y la motivación hacia el estudio, ofreciendo al estudiante herramientas suficientes para contrastar el cúmulo de información a su alcance. Esto es posible cuando se integran docencia e investigación, temática recurrente en foros y eventos de educación, lo cual motiva este trabajo, cuyo objetivo es reflexionar sobre el potencial que tiene el proceso investigativo en la enseñanza de la música. El análisis de documentos y los resultados de un debate grupal no estructurado propiciaron ofrecer alternativas para el aprovechamiento de las potencialidades del proceso investigativo en la enseñanza de la música.
\end{abstract}

Proceso investigativo, Alternativas, Enseñanza de la música

\begin{abstract}
Artistic education today involves a superior amount of effort compared to previous decades; with no intention of discrediting the quality and rigor exerted, in the past, student, group and teacher shared gradually ideas, resources, techniques and emotions inside and outside the classroom. Currently, the process is accelerating; the considerable amount of research on, about and for the sake of art, along with the technological impact and the existence of social networks that facilitate sharing, stimulates information inequalities, which assign new challenges to the teacher: to foster creativity and motivation towards studying, while offering the student suitable tools to assimilate the available set of information. This is possible when teaching and research are integrated, a recurring theme in forums and educational events, which motivates the objective of this work, which is to consider the potential of the research process in music teaching. An analysis of documents and the results of a non-structured group debate offered alternatives to exploit the potential of the research process in music teaching.
\end{abstract}

Research process, Alternatives, Music teaching

Citación: JUAN-CARVAJAL, Mara Lioba, JUAN-CARVAJAL, Dargen Tania, SÁNCHEZ-USÓN, María José y VDOVINA, María. Alternativas para el aprovechamiento del proceso investigativo en la enseñanza de la música. Revista de Filosofía y Cotidianidad. 2019, 5-16: 10-19

\footnotetext{
$\dagger$ Investigador contribuyendo como primer Autor.
} 


\section{Introducción}

La profesión del docente en la educación superior está hoy permeada de influencias externas debido a la globalización y el acelerado desarrollo científico-técnico y social que, en materia de comunicación, impactan en el sistema. En consecuencia, se caracteriza por un dinamismo que conlleva un esfuerzo superior respecto a décadas anteriores; la organización por disciplinas, asignaturas, o módulos que reflejaban el entramado que develaba los rasgos de la profesión, unido a la especialización del profesor, aseguraban la calidad y el rigor del proceso de enseñanza-aprendizaje (en adelante proceso de enseñanza).

En la actualidad, el impacto tecnológico provoca discrepancias que exigen al profesor el conocimiento de nuevas herramientas que fomenten la creatividad y promuevan la motivación al estudio, incluso guiando al discípulo hacia el examen de las referencias que origina tal disconformidad para comparar y tomar decisiones.

El empleo de la tecnología en la enseñanza es cada vez mayor y más exigente; la tendencia se encamina hacia la disposición tecnológica en cualquiera de sus variantes: tecnología del consumidor -herramientas creadas con fines recreativos o profesionales-; estrategias digitales -para el perfeccionamiento del proceso de enseñanza-aprendizaje-; tecnologías en internet -potencial a disposición de todos, herramientas en red que complementan el proceso-; tecnologías educativas -diseñadas específicamente para la docencia-; o tecnologías en redes sociales (Johnson, Estrada, \& Freeman, 2014)- su uso, cada vez más generalizado en la sociedad dada la facilidad de intercambio, impacta en gran medida en las clases.

La enseñanza artística, como todo proceso pedagógico, tiene infinitas posibilidades proporcionadas por las bondades que ofrecen las tecnologías de la informática y las comunicaciones; el discurso al respecto permite identificar una serie de beneficios para la educación superior:
Acceso fácil a la información, variabilidad disponible, elevados parámetros de rapidez durante su procesamiento, eliminación de barreras espacio-temporales, posibilidad de retroalimentación e interactividad, creación de espacios flexibles de aprendizaje, optimización de las actividades docente-investigativas, y celeridad en la gestión de procesos (Gómez, Contreras, \& Gutiérrez, 2016), lo que se aplica a todas las ramas del arte, dentro de ellas a la música.

La facilidad de viajar por el tiempo a través de grabaciones reales, o por medio de simulaciones a partir de una base de datos que caracterice el momento que se recrea, ofrece a la enseñanza artística la observación, escucha, comparación y progresión en la imaginación; elementos motivadores para la creación que, acompañados de un sustento teórico, facilitan dinamizar el proceso docente y aprovechar el gran espectro de información a disposición del estudiante.

Sin lugar a dudas la explotación de este potencial informativo depende en gran medida de la preparación del docente; la multidisciplinariedad en la gestión, unida a la estimulación del estudiante, demanda elevar la maestría pedagógica del profesor o tutor para la formación del músico; es decir, apropiarse de una cultura general que procure al estudiante elementos comparativos en base a indicadores relativos a la territorialidad, tradición, época, formas o métodos, de manera que éste desarrolle las competencias necesarias para el ejercicio profesional; así mismo, el docente identificará problemas, vías de solución y experiencias acordes con las particularidades de la rama del arte a la que tribute, para gestionar su clase.

Estos procedimientos se estiman en el saber y querer hacer: cultivar el empleo de las bondades del proceso investigativo para impactar en la calidad de las clases. La modelación del contenido declarado en los programas de estudios, unida al conocimiento de las particularidades socio-económicas, de lo histórico, lo tradicional, lo clásico y lo popular, confiere una visión de lo antiguo y de cómo ha evolucionado hacia lo actual. Es ahí donde existe un caudal de información al alcance del profesor para la integración de los procesos docente e investigativo, a fin de que, en consecuencia, se logre un impacto positivo en la enseñanza artística. 
La investigación en artes, cambia el sentido de percepción, transforma al investigador de manera que le es imposible volver a escuchar, criticar o ejecutar una obra artística sin un estudio que abarque la universalidad de lo que en ella se pueda apreciar. (Sánchez-Usón, Juan-Carvajal, Vdovina, \& Juan-Carvajal, 2016, pág. 102).

Es precisamente esa universalidad la que el profesor de música debe detonar en las clases para motivar la búsqueda y la investigación, aspecto que el docente puede emplear en beneficio del estudiante y de sí mismo como un sistema de retroalimentación.

La motivación tiene como punto de partida las necesidades del individuo. El docente, en su accionar, debe provocar otras nuevas, aprovechar las potencialidades del contexto y tener identificadas las características de los estudiantes para desarrollar procesos emocionales y cognitivos que generen la necesidad de enfrentar lo desconocido sin temor al fracaso y con el fin de alcanzar el éxito.

Particularmente en la enseñanza de un instrumento musical el trabajo diferenciado con los alumnos para alcanzar esta meta tiene mayores posibilidades; en esta profesión, el docente se convierte en un tutor que acompaña al estudiante no sólo en la mayor parte de su carrera, sino en toda ella, lo cual facilita la caracterización del discente y el paso de lo instrumental a lo espiritual.

El profesor de arte debe lograr mantener latente las ansias por conocer y por entregar, dado que el artista se revela al público y la disposición en esa entrega le permite conseguir sus propósitos. No es tarea fácil la caracterización del estudiante; ésta implica indagar en los rasgos de su personalidad, la cual constituye un entramado complejo que define la subjetividad humana y se manifiesta en " (...) la conducta, el comportamiento y las expresiones verbales (...)”. (Fernández, 2005, pág. 77).

En consecuencia, la observación pedagógica debe estar presente en toda actividad docente para no perder de vista los detalles que indican las potencialidades de trabajo con cada estudiante, dotándolo de herramientas metodológicas para orientarse hacia el éxito, de modo que adquiera confianza en el alcance de las metas propuestas.
Una alternativa en este sentido es la atención y promulgación de experiencias positivas anteriores, tanto propias como ajenas: el apoyo moral sobre la narración de anécdotas, el estímulo basado en los objetivos alcanzados anteriormente, o el ofrecimiento de una visión basada en la posibilidad de transitar lo proyectado hasta conquistar las metas, hacen que una situación frustrante sea factible de rediseño y se ponga mayor empeño en lograr el triunfo. Un profesor que sea capaz de integrar los procesos docente e investigativo y aprovechar el impacto que este último puede tener en la enseñanza, posee mayores posibilidades de superación con sus estudiantes.

Determinar el impacto del proceso investigativo en el aula tiene diversos puntos de vista; por lo general, la denominada "medición de impacto" transita por la comparación de momentos a partir de indicadores específicos, tales como el cumplimiento de objetivos, enjuiciamiento de la valía del proceso o la emisión de un juicio de valor que facilite la toma de decisiones. Sin embargo, no siempre el impacto está relacionado con alcanzar una meta, su acción estará latente durante todo el proceso investigativo:

(...) la valoración del impacto se puede realizar en cualquier momento de la investigación y precisan que en las primeras etapas se valoran causas y efectos, también enfatizan en estimar los cambios generados en condiciones concretas a partir de la medición de variables definidas como impacto. (Estacio \& et.al, 2009).

Es notorio el criterio de autores que ven en la evaluación de impacto un proceso conductual, cognitivo, motivacional $y$ emocional, el cual promueve la satisfacción de necesidades individuales impuestas por una cultura organizacional:

El índice de impacto actúa como mercancía de intercambio entre investigador y organización universitaria para satisfacer una necesidad individual, organizacionalmente impuesta. La lucha por el índice de impacto produce artículos como mercancía, pues se convierten en trabajo productivo y le añaden valor. (Santos \& Fernández-Ríos, 2016, pág. 42). 
Por otro lado, el impacto es visto de diversas maneras no obstante el criterio recurrente del cambio y su influencia en la toma de decisiones. En su medición se precisan cuatro ventajas potenciales: efecto demostrativo dado por el conocimiento del estado del resultado investigativo o innovador; efecto orientador, por la necesidad de tener una visión cosmopolita del contexto y una clara determinación de las potencialidades del objeto; efecto motivador, ya que impulsa a la innovación, y efecto integrador, visto como el papel de centralización que juega en un sistema de ciencia e innovación tecnológica. El principal objetivo en la medición de impacto "(...) es evaluar el beneficio tangible, la repercusión del resultado y no el resultado en sí mismo (...)". (Quevedo, Chía, \& Rodríguez, 2002, pág. 10).

Evaluar el impacto no es precisamente el objetivo propuesto en este trabajo; la idea versa sobre la utilidad de los resultados de la aplicación de métodos de investigación a fin de reflexionar sobre el potencial que tiene el proceso investigativo en la enseñanza de la música. Por consiguiente, se ofrecen alternativas para el aprovechamiento de las potencialidades del proceso investigativo en la enseñanza de la música.

\section{Desarrollo de una idea}

De acuerdo con las diversas fuentes bibliográficas consultadas, el primer momento del proceso investigativo es la generación de la "idea" que, sin precisión alguna, permite visualizar una meta e identificar dentro de un contexto un sinnúmero de problemáticas que inciden en el correcto funcionamiento del objeto que se estudia.

Es esa visión la que induce la búsqueda de elementos que caractericen y enmarquen la problemática identificada; por ejemplo, en la enseñanza de la música, existe un amplio espectro de obras para los diversos instrumentos en diferentes formatos que han sido acumuladas por la humanidad; sin embargo, en las instituciones docentes, no siempre se cuenta con los títulos (partituras) o referencias sobre autores representativos de un medio determinado, lo que pudiera ser objeto de motivación para el estudio. ¿Por qué no aprovechar en las clases los resultados de procesos investigativos que compilan tal información?
(...) en la educación universitaria avanzada, el genio de la investigación está en todas partes fuera de la botella. Si bien la preparación para el trabajo de investigación se basa en la investigación, la preparación para la práctica profesional se conforma cada vez más a partir de la investigación (...). (Clark, 1997, págs. 380-381).

Existen investigaciones que promueven el estudio de casos o la historia de vida como métodos científicos para descubrir las características de una época, obra o un autor determinado, y en sus aportaciones se develan rasgos referentes al contexto, momento histórico, personalidad, importancia social, influencia de otras latitudes o épocas, o recursos tecnológicos existentes.

Todo ello permite al estudiante, particularmente en la enseñanza de un instrumento, comprender por qué una composición musical se diferencia de otra, por qué en la forma, la armonía y demás elementos constitutivos de la música se manifiestan diferentes orientaciones simbólicas que en correspondencia con su enlace o disposición revelan particularidades de una corriente o tendencia en una época dada, o por qué los intérpretes le imprimen un sello personal a la ejecución de una obra musical.

Es evidente que estas no serían preguntas científicas a realizar durante una investigación para alcanzar un título académico o grado científico, pero los resultados que se obtengan justifican su consideración para formularlas, a fin de estimular a los estudiantes en la búsqueda de información orientada hacia el programa de estudio correspondiente a su currículo. Ello proporcionará, además de mayor cultura, expresar el sentido espiritual que debe acompañar a una interpretación musical para que llegue al público.

Es preciso significar que conforme con el currículo para la formación del músico, el nivel en que se encuentre y la disciplina o asignatura que curse será la temática a trabajar y la selección de las obras o compositores. No obstante, se logrará mayor efecto si ésta se corresponde con la caracterización que se haya realizado de los estudiantes. 
El docente que investiga transforma el proceso de enseñanza-aprendizaje, cambia su dinámica, la intención en la lectura, la interpretación, la comprensión del lenguaje y su proyección; se abre un abanico de saberes que facilitan el análisis, la contrastación, el intercambio, los métodos de enseñanzaaprendizaje y la motivación a la escucha de obras musicales con características parecidas a las estudiadas.

\section{(...) El lenguaje musical no pretende sino} la comunicación a través de una propuesta variada, una apertura de códigos en los que el compositor se regocija en mostrar su propia personalidad y decir lo que le es dado de manera auténtica. Es así como el entendimiento de la obra musical sólo puede darse a través de un análisis sociohistórico y cultural del individuo, su entorno y su campo de acción. (JuanCarvajal \& Juan-Carvajal, 2014).

Esta posición implica la presencia de cierta complicidad entre el profesor de música y el estudiante, dado que en el proceso de formación no sólo se incide en el aspecto instrumental o técnico, sino que despierta lo espiritual, y el análisis de los cambios de estilo, en correspondencia con la época y el contexto, brinda las herramientas para la comprensión de las variaciones en la armonía y la notación musical. Se desarrolla un sentido estético en el que afloran o se valoran juicios, tendencias, técnica, características, rasgos personales y emociones, que denotan sentimientos y sensaciones presentes en la traducción de los signos grabados en una partitura, durante la ejecución de una obra musical.

(...) La sensación que se produce al contemplar, al escuchar o al trasmitir una interpretación crea un estado inexplicable por la falta de comunicación entre el lenguaje sugerente (sígnico) y el lenguaje hablado con que nos comunicamos. Es así como consciente o inconscientemente estamos buscando la explicación de las sensaciones. (Vdovina, 2010, pág. 65).

Durante el proceso investigativo se ubican y clasifican las fuentes de información; lo que proporciona el acceso de acuerdo con lo proyectado, el diseño de espacios de reflexión, lecturas de informes.
La caracterización y ejecución de las obras (o partes de ellas) en las que se manifiesta la temática investigada, a fin de que la búsqueda sea factible y rigurosa.

El estudio del binomio enseñanzaaprendizaje durante un período más o menos corto propicia la identificación de los procesos o técnicas que ofrecen mayor grado de dificultad al estudiante. Una experiencia positiva es fomentar el debate acerca de los resultados alcanzados por los estudiantes de un mismo nivel; el cual podrá estar intencionado hacia los aspectos que presentan cierta complicación. Este puede dividirse por temáticas según las características del estudiantado o del compositor seleccionado, las cuales deben quedar claras para el docente desde el momento de preparación de la actividad (Figura 1).

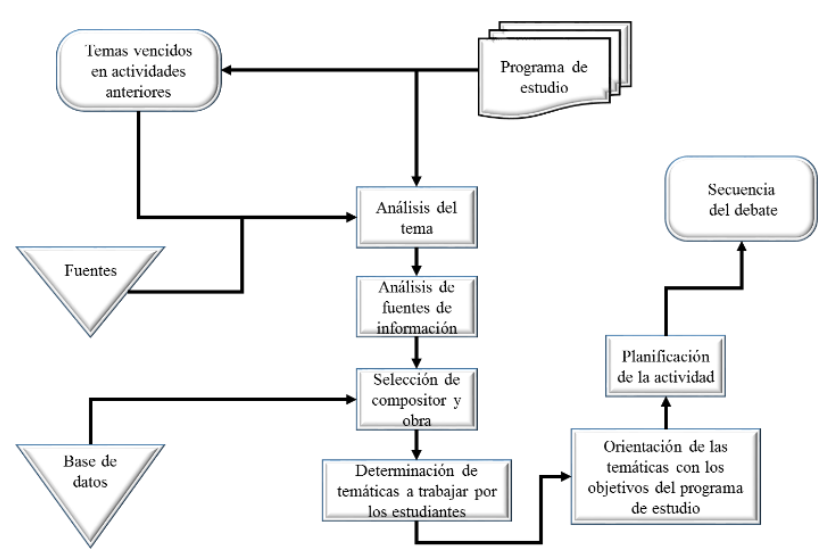

Figura 1 Proceso de preparación del debate, selección de temáticas

Fuente: Elaboración Propia

Durante la investigación afloran características desconocidas del proceso de creación de una obra musical; éstas pueden estar relacionadas con el compositor, el entorno en que se creó o con el momento histórico en que se generó; por ejemplo, el análisis temporal de la vida de un compositor dado ofrece información sobre las creaciones en un período, la influencia que tuvo de otros compositores, el contexto, el momento en que se formó y desarrolló su trabajo, o si paralelamente, se desempeñaba en otras esferas, como la dirección orquestal, la interpretación (ejecución de algún instrumento musical) y/o la docencia; también, puede revelar los procesos de transformación en la composición o en los instrumentos musicales para los cuales compuso, todo lo cual puede utilizarse en las disciplinas teóricas de la enseñanza de la música. 


\section{Beneficios de los resultados de una investigación para la enseñanza de la música}

Durante el estudio se combinaron los métodos, análisis de documentos y discusión grupal no estructurada, a fin de identificar alternativas en el proceso investigativo para la enseñanza de la música; con este objetivo se realizó el análisis de artículos relacionados con los procesos investigativo y pedagógico en la formación del profesional de la música, cuyos resultados ofrecieron los métodos más empleados para el estudio de un compositor y su obra musical, entre los que se distinguen el analítico-sintético y la historia de vida. Con la discusión grupal no estructurada, cuyo tema se centró en la utilización de los resultados de las investigaciones en la enseñanza de la música, se acordaron alternativas a emplear dado el potencial de ambos procesos.

Una estrategia metodológica para el análisis de los resultados del método analíticosintético puede apreciarse en la Figura 2; el esquema facilita el examen de las temáticas dirigidas a los aspectos relacionados con los objetivos definidos en el programa de estudio para la formación del instrumentista, en nuestro caso, de las cuerdas frotadas; las tendencias compositivas de acuerdo con las etapas estudiadas y los territorios, así como las peculiaridades en las armonías, elementos rítmicos, melodías y estructuras y formas según las características de las obras y la época en que se crearon, lo cual permite el diseño de las tareas docentes de acuerdo al nivel de los estudiantes y los objetivos del año en curso.

Significativo es estimular el estudio multidisciplinario y el desarrollo de valores en los estudiantes a partir del análisis del contexto en que se formó y desenvolvió el compositor, las particularidades de la época y el impacto que ésta tiene en la obra. Este análisis contribuye, además, al incremento de la cultura general, al respeto por la cultura organizacional de la institución a la que pertenecen y al incremento de la responsabilidad con su formación.

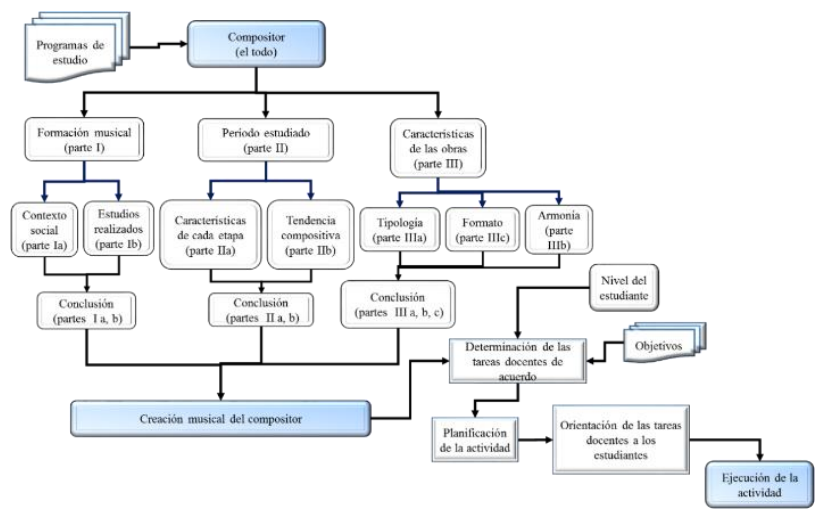

Figura 2 Proyección del resultado de la aplicación del método de analítico-sintético aplicado al estudio de un compositor

Fuente: Elaboración Propia

El desglose de las tareas docentes, paralelo a las etapas en la estructura del método seleccionado para la investigación, favorece la motivación de los estudiantes; se sienten capaces de vencer cada meta ya que, a pesar de ser tensa y rigurosa, se dosifica de acuerdo con las actividades programadas y a sus posibilidades de ejecución.

La coordinación entre los aspectos tecnológicos, instrumentales, históricos, y afectivos, necesarios para la formación del artista -dado las particularidades de su profesión donde el eje principal es la aceptación del público-, tiene mayor posibilidad de alcance cuando se combinan y contrastan los resultados de diferentes métodos y técnicas de investigación. Complementar el método analítico-sintético con la historia de vida contribuye a la caracterización del compositor y al incremento, dosificación y diversificación de actividades con los estudiantes.

La historia de vida, como toda investigación de tipo cualitativo, tiene un fuerte componente interpretativo; dar cumplimiento a su estrategia metodológica depende en gran medida de la situación y la posibilidad de recogida de datos. Como método investigativo, al referir el "(...) modo cómo se relaciona el sujeto de investigación con su objeto, en correspondencia con los niveles del conocimiento (teórico o empírico) a partir de la práctica y de la valoración del objeto" (Martínez, 2003, pág. 120), sustenta que la realidad se devela a través de disquisiciones individuales o colectivas. 
El efecto a proyectar puede encaminarse al entendimiento del fenómeno desde la posición $\mathrm{y}$ vivencias de los entrevistados: el propio compositor, musicólogos, otros compositores, intérpretes, docentes y directores de orquesta.

La información que se obtiene con el empleo de este método está permeada de extensas descripciones, por lo que es recomendable el empleo de herramientas tecnológicas para la recogida, registro y discriminación de datos.

El método historia de vida “(...) toma en consideración el significado afectivo que tienen las cosas, situaciones, experiencias, y relaciones que afectan a las personas (...)". (Chárriez, 2012, pág. 51); su flexibilidad y posibilidad de representar el objeto según se describe permiten el estudio del objeto que se investiga en su conjunto, aspecto que le imprime cierto grado de complejidad.

Considera y promueve la comunicación abierta y horizontal con los sujetos que participan, lo cual facilita la descripción de fenómenos según las memorias de los protagonistas $\mathrm{o}$ expertos. $\mathrm{Su}$ estrategia metodológica integra al análisis de documentos y la entrevista.

El análisis de documentos, como parte de la historia de vida, consiste en la exploración de cualquier objeto de registro que contenga la información del compositor a estudiar -ya sea en formato impreso o digital-. Su estrategia metodológica (Figura 3) ofrece espacios que agilizan la realización de diversas actividades con los estudiantes a partir de los resultados obtenidos en cada momento.

Los documentos pueden ser primarios (contienen la información básica) o secundarios (complementan los primarios); durante el período de búsqueda bibliográfica exploratoria no debe discriminarse ningún documento que tenga relación con la temática investigada, y se recomienda destacar en la ficha técnica su ubicación.

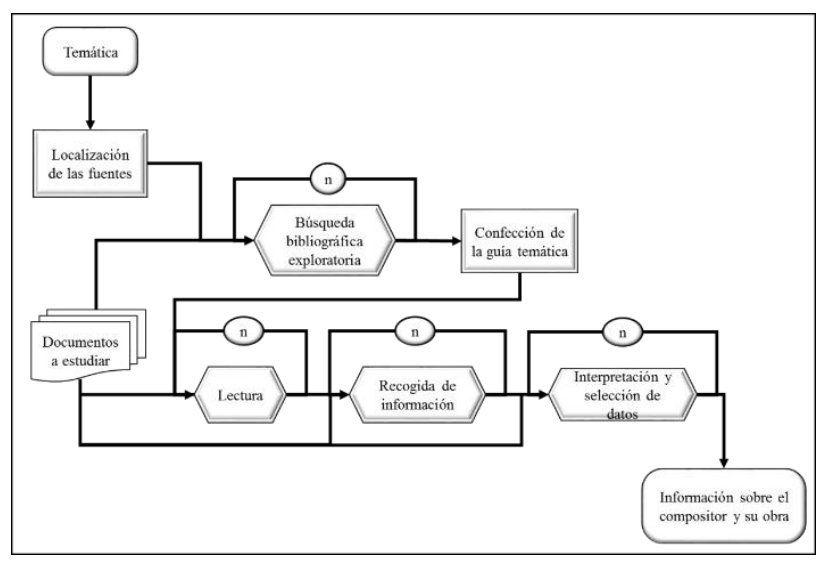

Figura 3 Diagrama de flujo: estrategia metodológica del análisis de documento

Fuente: Elaborado por las autoras, sustentado en la propuesta de Alonso \& Saladrigas (2002)

Una alternativa para el diseño de las actividades no lectivas a realizar con los estudiantes es ponderar el impacto de los resultados que favorezcan la motivación y la creatividad. La creatividad se puede apreciar en la manifestación o disposición con que los educandos participen tanto de la actividad lectiva como en la búsqueda y recogida de información. Significativo es observar la integración y puesta en marcha de sus capacidades, las que se distinguen, según Mitjans (1991), en correspondencia con las tendencias motivacionales que les impulsen a la acción.

Los momentos de lectura, recogida de información e interpretación y selección de datos, facilitan la visión multidisciplinaria del proceso de enseñanza, para lo cual el docente ha de brindar una selección temática que abarque parte del abanico de disciplinas y asignaturas cursadas por el estudiante. La determinación de indicadores para su aplicación se realiza teniendo en cuenta el nivel en que se encuentren los educandos y los objetivos en los programas de estudio, de tal manera que los resultados de las investigaciones impacten positivamente en la formación del músico.

La posibilidad de comunicación abierta e intencionada en las actividades a partir de las propuestas de debate incrementa la motivación de los estudiantes hacia la búsqueda y comparación entre nuevas fuentes. La entrevista, como complemento de la historia de vida, enriquece las valoraciones teóricas realizadas, brinda la posibilidad de precisar detalles, a partir de la visión del entrevistado, y promueve el estudio. 
Puede realizarse de manera individual o en pequeños grupos y su formato depende del objetivo que persiga el investigador. Por la particularidad de estar en diálogo directo con el interlocutor, facilita la atención al lenguaje verbal y extraverbal.

Su lógica de aplicación parte de la definición del objetivo y la determinación de los aspectos sobre los que se necesita información. $\mathrm{Su}$ preparación demanda la formulación de temáticas a tratar -orientación que se brinda con anticipación- y una exploración previa para conducirla con dinamismo, a fin de no perder el interés del entrevistado. Para su realización se prepara el local asegurando la organización, un clima favorable y comodidad de los sujetos que intervienen, cuidando tener las herramientas de registro.

Un elemento esencial es la selección del o los informantes; se selecciona el que disponga del conocimiento y la experiencia, posea la habilidad para reflexionar y expresarse con claridad, y esté dispuesto a participar en el proceso investigativo.

El conocimiento del tema, prestigio tanto del entrevistado como del compositor estudiado- e impacto social, pueden ser explotados por el docente para elevar la motivación y la calidad en la interpretación de una obra musical, así como la realización de informes creativos o productos audiovisuales donde se destaquen las características de la obra, del compositor, o las particularidades del medio y de la institución docente donde cursa estudios.

En general, la historia de vida viabiliza la construcción de modelos durante las diferentes fases de una investigación. La facilidad de recoger la información, inclusive de los protagonistas de los hechos, y la contrastación de los datos registrados validan la sistematización de los resultados durante la formación del músico.

El potencial de las fuentes empleadas permite el diseño de talleres donde se fomente el intercambio entre los protagonistas de los hechos, los especialistas o expertos y los estudiantes, lo cual suscita el interés por superarse durante su actuación, ya sea en clases frente al profesor o tutor, o frente al público.
En correspondencia con los resultados de la aplicación de los métodos de investigación se pueden diseñar actividades presenciales o no (dado el impacto tecnológico en el proceso de enseñanza), tales como:

- Lectura comentada sobre las características de la obra y el impacto del contexto en que fue creada.

- Escucha e identificación de los rasgos característicos del género y de la época. Debate sobre el formato empleado y la posible influencia del entorno.

- Escucha, comparación e identificación de las características de la interpretación. Análisis de la partitura e identificación de las particularidades del género.

Concierto - debate, con la participación del compositor o especialistas, acerca de la sonoridad y posibilidades del instrumento.

- $\quad$ Análisis de la obra e identificación del desarrollo compositivo de su autor.

Como alternativas, para el aprovechamiento del potencial del proceso investigativo en la enseñanza de la música, de la entrevista grupal no estructurada resultaron:

- Diseño de tareas docentes utilizando compilaciones y/o productos audiovisuales que pueden agruparse de acuerdo con el nivel del estudiante, el instrumento musical, las disciplinas o asignaturas, la época, el estilo, el género, el contexto, entre otros.

Planificación de talleres, debates grupales, o conciertos-debate (previa orientación a los estudiantes) donde se fomente la discusión y se caracterice al compositor o al contexto en que se escribió la obra musical; con o sin la presencia del compositor u otros expertos.

Orientación al estudio de obras musicales (grabadas o por asistencia a conciertos) a partir de indicadores ofrecidos a los estudiantes con anterioridad, relacionados con la calidad de la ejecución, la sutileza, o la impronta del intérprete.

- Realización del trabajo diferenciado a partir de la caracterización de los estudiantes y de la obra musical de acuerdo con las motivaciones, necesidades, entre otros. 
- Transformación de la dinámica del proceso de enseñanza de la música atendiendo al saber teórico-práctico disponible y a los fenómenos actuales provocados por el desarrollo tecnológico. Orientación al estudio del contexto y estilos según la época, para la comprensión de los cambios en la armonía, o la notación musical, entre otros.

Elaboración de bases de datos que ubiquen la fuente de información sobre compositores, obras musicales, o estudiosos de la música, entre otros, para facilitar la organización y rigurosidad del proceso de enseñanza.

- Análisis del proceso de enseñanza a partir de indicadores con los que se determinen los momentos, obras musicales, técnica o ejercicios de mayor dificultad para los estudiantes, lo cual facilita el diseño didáctico de la disciplina o asignatura atendiendo a las dificultades identificadas.

- Conversatorios donde se atienda, promueva o estimule a los estudiantes a partir de experiencias positivas en la interpretación de la obra musical y en correspondencia con los objetivos alcanzados.

El diseño de alternativas para el aprovechamiento del potencial del proceso investigativo estará en correspondencia con los objetivos de los programas de las disciplinas o asignaturas en la formación del músico; las características del estudiantado; el nivel que cursen; el potencial tecnológico disponible, y la creatividad del docente

\section{Conclusiones}

El desarrollo tecnológico alcanzado por la sociedad impacta en la educación superior con bondades referidas al amplio acceso y variabilidad de la información que, a su vez, generan contradicciones en el estudiante. Como consecuencia, se exige al profesor mejoras en su maestría pedagógica, con el fin de desarrollar un proceso pedagógico de calidad y el fomento de la creatividad y motivación hacia el estudio. Una alternativa para estas exigencias se presenta en el aprovechamiento del proceso investigativo.
El proceso de la investigación favorece la transformación y el dinamismo del proceso pedagógico; particularmente en lo referido al diseño de tareas y actividades, y a los métodos de enseñanza. El espectro de conocimientos teórico-práctico resultante facilita el análisis, la comparación, el intercambio, la escucha, la comprensión y promueve el incremento de la motivación por el estudio.

La aplicación de los resultados de los métodos de investigación favorece el diseño de tareas docentes en correspondencia con el nivel del estudiante y los objetivos proyectados, la dosificación y diversificación de las actividades, la elaboración de diseños didácticos, y el trabajo diferenciado con los estudiantes.

\section{Referencias}

Alonso, M., \& Saladrigas, H. (2002). Para investigar en comunicación social. Guía didáctica. La Habana: Félix Varela.

Chárriez, M. (Diciembre de 2012). Historias de vida: una metodología de investigación cualitativa. Revista Griog, 5(1), 50-67.

Clark, R. (1997). Las universidades modernas: espacios de investigación y docencia. (M. Á. Porrúa, Ed.) Coordinación de humanidades, UNAM.

Estacio, A., \& et.al. (2009). Evaluación de impacto de dos programas de formación del Sena: formación de técnicos y tecnológicos y formación especializada del recurso humano vinculado a las empresas. working paper No. 52, Centro de investigación económico y social. Recuperado el 3 de agosto de 2017, de http://www.repository.fedesarrollo.org.co/handl $\mathrm{e} / 11445 / 243$

Fernández, L. (2005). La personalidad. Algunos presupuestos para su comprensión. En Z. Fernández; \& Casals, J. C. Psicología General (págs. 76-90). La Habana: Félix Varela.

Gómez, M. E., Contreras, L., \& Gutiérrez, D. (mayo-agosto de 2016). El impacto de las tecnologías de la información y la comunicación en estudiantes de ciencias sociales: un estudio comparativo de dos universidades públicas. Innovación Educativa, 16(71), 61- 80. 
Johnson, L. A., Estrada, V., \& Freeman, A. (2014). NMC Horizon Report: 2014 Higher Education Edition. Texas: The New Media Consortium.

Juan-Carvajal, M. L., \& Juan-Carvajal, D. T. (2014). Cuerdas frotadas en Cuba. Medio siglo de creación. La Habana: CIDMUC.

Martínez, M.. (2003). Los métodos de investigación educacional: lo cuantitativo y lo cualitativo. En Martínez, M., et al. Metodología de la investigación educacional. Desafío y polémicas actuales (pp. 113-137). Ciudad de La Habana: Félix Varela.

Mitjans, A. (1991). La relación entre personalidad, creatividad y motivación. Implicaciones en la práctica educativa. Revista Cubana de Psicología, VIII (2-3), 117-125.

Quevedo, V., Chía, J., \& Rodríguez, A. (2002). Midiendo el impacto. Ciencia, Innovación y Desarrollo, 7(1), 8-13.

Sánchez-Usón, M. J., Juan-Carvajal, M. L., Vdovina, M., \& Juan-Carvajal, d. t. (octubrediciembre de 2016). Arte y ciencia, un debate inconcluso. ECORFAN. Revista Investigaciones Sociales, 2(6), 94-106.

Santos, M. A., \& Fernández-Ríos, L. (septiembre-diciembre de 2016). El factor de impacto y el futuro de las revistas académicas. El riesgo de patologización. Innovación Educativa, 16(72), 35-51.

Vdovina, M. (2010). La interpretación como proceso de creación. En G. C. (Coord.), Colorama musical: nuevos problemas, nuevas perspectivas. Zacatecas: Plaza y Valdés, 57-80. 